\title{
Voltage-Dependent T-Type Calcium Channel Subunit Alpha-1G
}

National Cancer Institute

\section{Source}

National Cancer Institute. Voltage-Dependent T-Type Calcium Channel Subunit Alpha-1G. NCI Thesaurus. Code C105858.

Voltage-dependent T-type calcium channel subunit alpha-1G (2377 aa, $262 \mathrm{kDa}$ ) is encoded by the human CACNA1G gene. This protein is involved in both membrane depolarization and calcium import. 